\title{
Biotin provisioning by horizontally transferred genes from bacteria confers animal fitness benefits
}

\author{
Fei-Rong Ren $\mathbb{D}^{1} \cdot$ Xiang Sun $\mathbb{D}^{1} \cdot$ Tian-Yu Wang $\mathbb{D}^{1} \cdot$ Ya-Lin Yao $\mathbb{D}^{1} \cdot$ Yan-Zhen Huang $\mathbb{D}^{1} \cdot$ Xue Zhang ${ }^{2} \cdot$ \\ Jun-Bo Luan $\mathbb{1}^{1}$
}

Received: 3 December 2019 / Revised: 1 June 2020 / Accepted: 11 June 2020 / Published online: 22 June 2020

(c) The Author(s), under exclusive licence to International Society for Microbial Ecology 2020

\begin{abstract}
Insect symbionts are widespread in nature and lateral gene transfer is prevalent in insect symbiosis. However, the function of horizontally transferred genes (HTGs) in insect symbiosis remains speculative, including the mechanism that enables insects to feed on plant phloem deficient in B vitamins. Previously, we found there is redundancy in biotin synthesis pathways from both whitefly Bemisia tabaci and symbiotic Hamiltonella due to the presence of whitefly HTGs. Here, we demonstrate that elimination of Hamiltonella decreased biotin levels but elevated the expression of horizontally transferred biotin genes in whiteflies. HTGs proteins exhibit specific expression patterns in specialized insect cells called bacteriocytes housing symbionts. Complementation with whitefly HTGs rescued E. coli biotin gene knockout mutants. Furthermore, silencing whitefly HTGs in Hamiltonella-infected whiteflies reduced biotin levels and hindered adult survival and fecundity, which was partially rescued by biotin supplementation. Each of horizontally transferred biotin genes are conserved in various laboratory cultures and species of whiteflies with geographically diverse distributions, which shares an evolutionary origin. We provide the first experimental evidence that biotin synthesized through acquired HTGs is important in whiteflies and may be as well in other animals. Our findings suggest that B vitamin provisioning in animal-microbe symbiosis frequently evolved from bacterial symbionts to animal hosts through horizontal gene transfer events. This study will also shed light on how the animal genomes evolve through functional transfer of genes with bacterial origin in the wider contexts of microbial ecology.
\end{abstract}

\section{Introduction}

Insects are a highly successful group of animals, some of which are able to utilize a wide range of nutrient-depleted food resources from plant leaves to animal blood [1-3]. One of the driving forces shaping insect adaption to diverse feeding habits are microbial symbionts, which are

These authors contributed equally: Fei-Rong Ren, Xiang Sun

Supplementary information The online version of this article (https:// doi.org/10.1038/s41396-020-0704-5) contains supplementary material, which is available to authorized users.

Jun-Bo Luan

jbluan@syau.edu.cn

1 Liaoning Key Laboratory of Economic and Applied Entomology, College of Plant Protection, Shenyang Agricultural University, Shenyang 110866, China

2 China Agricultural University, Beijing 100083, China widespread in nature $[4,5]$. Insects bear primary symbionts, which can provide their hosts with essential nutrients deficient in their food, and secondary symbionts that may confer ecologically important traits depending on the environments and physiological states [6]. Many of these symbionts are localized in the gut and hemocoel or within specialized insect cells called bacteriocytes [6]. Generally speaking, symbionts housed in bacteriocytes are vertically transmitted and evolve to have highly reduced genomes compared with free-living relatives [7]. Thus, bacteriocyte adaptation to extreme genomic degeneracy of symbionts is critical for maintaining insect symbiosis [7]. Although considerable progress has been made, studies on the bacteriocyte-symbiont interface remain in the early stages [7].

Horizontal gene transfer events have tremendous impacts on the genomic evolution of both prokaryotes and eukaryotes, and it has been known to occur very frequently in prokaryotes [8, 9]. However, an increasing number of horizontal gene transfer from bacteria to eukaryotes, 
especially in insects and mites, has been reported [9-11]. For example, it was demonstrated that horizontally transferred carotenoid desaturase or phytoene desaturase is responsible for carotenoid production in aphids and spider mites and influences body color $[12,13]$. In addition, horizontally transferred genes (HTGs) help insects to efficiently assimilate or detoxify plant produced metabolites [14, 15]. HTGs are widespread in insect symbiosis and they presumably function with other genes coded by the symbiont, making some metabolic pathways intact $[1,9,16,17]$. Recent studies have shown that the horizontally transferred RlpA4 with bacterial origin encodes a protein that is transported to an obligate endosymbiont in aphids [18], and silencing horizontally transferred $a m i D$ and $l d c A l$ in aphids reduces Buchnera abundance [19]. It has been demonstrated that the mealybug protein encoded by the HTGs of bacterial origin is transported into the cytoplasm of symbiont Moranella for peptidoglycan synthesis [20]. However, functional validation for most HTGs is lacking, so that the metabolic, biological, and evolutionary role of HTGs in insect symbiosis remains largely unknown.

B vitamins perform various important functions in animals [21]. Animals cannot synthesize these compounds so that acquire them from their diet [21]. Vertebrate animal blood and plant sap are deficient in B vitamins. How groups of insects that feed on these diets deal with nutritional deficiencies is of great interest. It has been experimentally demonstrated that bedbugs, ticks and tsetse flies that feed on animal blood are able to derive B vitamins from their symbionts [2, 22-27]. Insects feeding on plant phloem and xylem could also obtain B vitamins from symbionts, but this has been mainly inferred from their genomic data [28-33]. Few studies have experimentally examined the role of bacterial symbionts that supply B vitamins for herbivorous insects [34-36]. Therefore, how herbivorous insects cope with B vitamin deficiency in plant sap needs to clarified.

The whitefly Bemisia tabaci species MEAM1 is a widespread invasive pest in agriculture damaging a wide range of crops [37-39]. The MEAM1 whitefly species bears two symbionts, Portiera and Hamiltonella, in the same bacteriocyte, which is maternally inherited [40, 41]. Previously, we found three horizontally transferred bioA, bioD, and $b i o B$ were encoded in the genome of the whitefly $B$. tabaci MEAM1 using genomic and transcriptomic analysis $[17,42]$. Among them, bioB has acquired intron and all three genes are highly expressed in bacteriocytes as reported in the Planococcus citri mealybug [1, 17, 42]. These genes are presumably involved in biotin synthesis. However, the genome of the symbiont Hamiltonella in the whitefly also have biotin synthesis genes including bioA, bioD, and bioB $[17,42]$. Thus, whether whitefly HTGs and Hamiltonella contribute to biotin synthesis independently or collectively is unknown. In this study, the function of horizontally transferred biotin synthesis genes in the whitefly $B$. tabaci MEAM1-Hamiltonella symbiosis system was investigated by combining immunohistochemistry, complementation assays, gene silencing, biotin assay, insect performance observation, and evolutionary analysis. We reveal that although Hamiltonella can synthesize biotin, biotin provisioned by whitefly HTGs affects the survival and fecundity of adult whiteflies.

\section{Materials and methods}

\section{Insect rearing and plants}

The whitefly B. tabaci MEAM1 was cultured on cotton plants (Gossypium hirsutum, cv. Shiyuan 321) grown in compost supplemented with Miracle-Gro Water Soluble All Purpose Plant Food. Cotton plants were cultivated to the 6-7 true-leaf stage for use in experiments described below. For more details, see the Supplementary Text.

\section{PCR, quantitative PCR (qPCR) and qRT-PCR analysis}

The presence of Portiera, Hamiltonella and Rickettsia in the B. tabaci colony was identified by diagnostic PCR in combination with Sanger sequencing. Portiera, Hamiltonella, and Rickettsia were quantified by qPCR using the copy number of $16 \mathrm{~S} r R N A, 16 \mathrm{~S} r R N A$, and gltA genes, respectively, with the $B$. tabaci $\beta$-actin gene as internal standard for normalization. Relative gene expression was performed by qRT-PCR and calculated using the $\beta$-actin gene for transcript normalization in the symbiont elimination and gene silencing experiments. All primers used in this study are provided in Supplementary Table 1. Relative symbiont density and gene expression were calculated using the $2^{-\Delta \mathrm{Ct}}$ method [43]. For more details, see the Supplementary Text.

\section{Biotin measurement}

A microbiological assay was used for biotin quantification in whiteflies using Lactobacillus plantarum ATCC 8014 (Beijing Landbridge technology Limited, Beijing, China) referring to the protocol as described previously [44]. Briefly, in an experiment, 100 male and 100 female adult whiteflies within 1 week after emergence were collected using a mouth aspirator and flash-frozen in liquid $\mathrm{N}_{2}$. Insects from each treatment were pooled, weighed, and homogenized in citrate buffer using homogenizer MP FastPrep-24 (MP Biomedicals LLC, Santa Ana, USA) and incubated in sulphuric acid. Then samples were sterilized using a $0.2 \mu \mathrm{m}$ pore size filter (PALL Life Science, New York, USA), mixed with vitamin B7-deficient Difco biotin 
assay medium (thereafter called as B7DB; Becton, Dickinson and Company, USA) and inoculated with log-phase L. plantarum ATCC 8014. Cultures were incubated for $22-28 \mathrm{~h}$ at $37^{\circ} \mathrm{C}$. Standard concentrations of biotin (0-0.8 ng/mL; Sigma-Aldrich, St. Louis, MO USA) were mixed with the $L$. plantarum culture to create a standard curve. A negative control consisting of citrate buffer only was subjected to the complete procedure to ensure the retention of the initial biotin and a lack of additional biotin, respectively. Moreover, an additional negative control included only the assay medium lacking $L$. plantarum to exclude the contamination by other bacteria. The growth of $L$. plantarum was measured using a microplate reader (Versa Max Molecular Devices, Silicon Valley, USA) taking absorbance readings at $630 \mathrm{~nm}$. Biotin in whiteflies was quantified using the standard curve and normalized to the weight of insects included in the homogenate. For more details, see the Supplementary Text.

\section{Effects of Hamiltonella elimination by antibiotic treatment on whitefly biotin gene expression and biotin levels}

Hamiltonella was eliminated by treating whitefly adults with a cocktail of antibiotics [45]. Hundreds of adult whiteflies within 0-7 days post emergence (F0) were fed a $25 \%$ (w/v) sucrose solution supplemented with $500 \mu \mathrm{g} / \mathrm{mL}$ each of 3 antibiotics (i.e., ampicillin, gentamycin, and cefotaxime) (BBI Life Sciences, Shanghai, China), for 4 days. The artificial diets with antibiotics were renewed every 2 days. Control insects were fed only a sucrose solution that was not supplemented with antibiotics. Following the diet treatments, whiteflies were transferred to cotton plants and allowed to lay eggs. Female and male adult whiteflies were collected within 0-7 days after emergence at the F1 stage. DNA was extracted from 8 female adult whiteflies and used for symbiont quantification by qPCR. RNA was extracted from 25 male and 25 female adult whiteflies from each of the 3 biological replicates and used to compare the expression levels of horizontally transferred bioA, bioD, and bioB in Hamiltonella-cured and Hamiltonella-infected whiteflies by qRT-PCR. Biotin was extracted from 100 male and 100 female adult whiteflies for each of the 3 biological replicates and quantified in Hamiltonella-cured and Hamiltonella-infected whiteflies by microbiological assay as described above.

To see a time series of biotin amounts over time after antibiotic treatment, Hamiltonella was eliminated as described above and biotin was quantified in 100 male and 100 female adults of Hamiltonella-cured and Hamiltonellainfected whiteflies at day 5, day 10, and day 15 after emergence at the F1 stage. Three biological replicates were conducted.
Fluorescence in situ hybridization (FISH)

Localization of Portiera, Hamiltonella, and Rickettsia in the whole body of Hamiltonella-cured and Hamiltonellainfected adult whiteflies and Portiera and Hamiltonella in the bacteriocytes of Hamiltonella-infected adult whiteflies was investigated by FISH following the protocol as described previously [46]. For more details, see the Supplementary Text.

\section{Recombinant enzyme generation, antibody preparation, and western blot}

The open reading frame of whitefly bioA, bioD, and $b i o B$ was cloned and recombinant enzymes were generated. Custom-made polyclonal antibodies against BioA (predicted size, $49 \mathrm{kDa}$ ), BioD (predicted size, $24 \mathrm{kDa}$ ), and $\mathrm{BioB}$ (predicted size, $38 \mathrm{kDa}$ ) proteins were produced by ProbeGene Life Sciences Co. Ltd. following previously described methods $[18,47]$. The specificity of polyclonal antibodies was verified by western blot. For more details, see the Supplementary Text.

\section{Immunofluorescence microscopy}

Bacteriocytes and guts from adult female whiteflies infected with Hamiltonella and bacteriocytes from Hamiltonellainfected and Hamiltonella-cured adult female whiteflies at 7 days after emergence were dissected. Samples were fixed, permeabilized, and incubated with antibodies. Images were collected and analyzed on an FV3000 confocal microscope (Olympus, Japan). For more details, see the Supplementary Text.

\section{Functional complementation of $E$. coli biotin auxotrophs with whitefly HTGs}

To examine the metabolic function of horizontally transferred bioA, bioD, and bioB, the kanamycin resistance site was amplified from E. coli G11 using knockout primers (Supplementary Table 1). Then pKD46 plasmids expressing Lambda Red recombinase were transformed into the $E$. coli K-12 BW25113 by electroporation. Subsequently, the $E$. coli K-12 BW25113 bioA, bioD, and bioB knockout mutants (i.e., $-\Delta b i o A,-\Delta b i o D$, and $-\Delta b i o B$ ) were generated following the Lambda Red protocol as described previously [48-50]. E. coli mutants were screened using an LB media agar plate with $0.1 \mathrm{mg} / \mathrm{mL}$ kanamycin. PCR amplification was carried out using primers specific for deleted genes, the kanamycin resistance site and flanking locus to verify the loss of the parental fragment and gain of the kanamycin resistance site (Supplementary Table 1). The E. coli mutants were then made into competent cells by electroporation. For 
the functional complement experiments, E. coli K-12 mutant cells were transformed with the plasmids pMD19T-bioA, pMD19-T-bioD, pMD19-T-bioB, and pMD19-T empty vector (negative control), respectively, following the previous methods [34]. The E. coli wild-type K-12, mutant $\mathrm{K}-12$, and mutant $\mathrm{K}-12$ transformants were grown overnight in LB agar media with $0.1 \mathrm{mg} / \mathrm{mL}$ ampicilin at $37^{\circ} \mathrm{C}$. The mutant K-12 transformants were verified by PCR amplification using primers specific for inserted genes. Then $E$. coli wild-type $\mathrm{K}-12$, mutant $\mathrm{K}-12$, and mutant $\mathrm{K}-12$ transformants were grown overnight in LB media and vitamin B7-deficient Difco biotin assay media at $37{ }^{\circ} \mathrm{C}$. All E. coli cells were washed twice in sterile distilled water and resuspended to measure the cell density at $\mathrm{OD}_{600}$ using a microplate reader (Versa Max Molecular Devices, Silicon Valley, USA). Finally, aliquots ( $2 \mu \mathrm{L}$ each) of cell suspensions were plated on LB media agar plates to assess cell growth after overnight incubation at $37^{\circ} \mathrm{C}$.

\section{dsRNA preparation}

dsRNAs specific to whitefly bioA, bioD, and $b i o B$ and GFP were synthesized using a T7 RiboMAX ${ }^{\mathrm{TM}}$ Express RNAi System kit (Promega, USA), following the manufacturer's instructions. For more details, see the Supplementary Text.

\section{Effects of silencing horizontally transferred biotin genes on biotin levels and whitefly performance}

To investigate whether silencing of horizontally transferred biotin genes influences biotin levels, 400 male and 400 female adult whiteflies infected with Hamiltonella within 1 week after emergence were fed $30 \%(\mathrm{w} / \mathrm{v})$ sucrose solution supplemented with dsGFP, dsbioA, dsbioD, or dsbioB, each at the concentration of $0.5 \mu \mathrm{g} / \mu \mathrm{L}$ for each biological replicate, for 3 days. ds $G F P$ serves as a control. RNA was extracted from 25 male and 25 female adult whiteflies to examine the expression of bioA at day 1 and 3 using 6 biological replicates or bioD at day 2 and 3 and $b i o B$ at day 1 and 3 using 3 biological replicates after dsRNA treatment. To examine whether silencing of whitefly biotin genes affects protein expression levels in bacteriocytes, whitefly bacteriocytes were dissected, fixed, permeabilized, and incubated with antibodies as described above. Images were collected and analyzed on an FV3000 confocal microscope (Olympus, Japan). In parallel, 100 male and 100 female adult whiteflies at day 3 in each biological replicate after dsRNA treatment were collected for biotin analysis as described above.

To measure the effect of knockdown of horizontally transferred biotin genes on whitefly survivorship, 150 female adult whiteflies infected with Hamiltonella within 1 week after emergence were fed $30 \%$ (w/v) sucrose solution supplemented with dsGFP, dsbioA, dsbioD, or dsbioB, each at the concentration of $0.5 \mu \mathrm{g} / \mu \mathrm{L}$, for 3 days. Mortalities of female whiteflies after 1,2 , and 3 days were recorded for each of the 3 biological replicates. To measure the effect of knockdown of horizontally transferred biotin genes on whitefly fecundity, 50 male and 50 female adult whiteflies within 1 week after emergence were fed $30 \%$ (w/v) sucrose solution supplemented with dsGFP, dsbioA, $\mathrm{ds} b i o D$, or dsbioB, each at the concentration of $0.5 \mu \mathrm{g} / \mu \mathrm{L}$, for 3 days. Subsequently, one female adult whitefly was released into a clip-cage attached to a cotton plant and allowed to lay eggs with $12,12,8$, and 10 replicates for the treatment with dsGFP, dsbioA, dsbioD, and dsbioB, respectively. After 7 days, egg numbers were recorded. RNA was extracted from adult whiteflies to verify the expression of bioA, bioD, and bioB.

To detect whether silencing of whitefly biotin genes influences the abundance of Hamiltonella, 40 female adult whiteflies infected with Hamiltonella within 1 week after emergence were fed $30 \%(\mathrm{w} / \mathrm{v})$ sucrose solution supplemented with ds $G F P$, dsbioA, dsbioD or dsbioB, each at the concentration of $0.5 \mu \mathrm{g} / \mu \mathrm{L}$. ds $G F P$ serves as a control. After feeding on dsRNAs for 3 days, whiteflies were collected. DNA was extracted from 8 dsRNA-fed female adult whiteflies and used for Hamiltonella quantification by qPCR as described above.

\section{Effects of biotin supplementation on whitefly performance}

To test whether biotin supplementation restores the survival of dsRNA-fed whiteflies, 150 female adult whiteflies within 1 week after emergence were fed $30 \%(\mathrm{w} / \mathrm{v})$ sucrose solution supplemented with dsGFP, dsbioA or dsbioA plus biotin, each at a final concentration of $0.5 \mu \mathrm{g} / \mu \mathrm{L}$ for dsRNA and $167 \mathrm{ng} / \mathrm{mL}$ for biotin for 3 days. Mortalities of female whiteflies after 1, 2, and 3 days were recorded for each of the 3 biological replicates.

To investigate whether biotin supplementation restores the fecundity of dsRNA-fed whiteflies, $\sim 50$ male and 50 female adult whiteflies within 1 week after emergence were fed $30 \%$ (w/v) sucrose solution supplemented with dsGFP, $\mathrm{ds} b i o A$ or dsbioA plus biotin, each at a final concentration of $0.5 \mu \mathrm{g} / \mu \mathrm{L}$ for dsRNA and $167 \mathrm{ng} / \mathrm{mL}$ for biotin for 3 days. Subsequently, one female adult whitefly was released into a clip-cage attached to a cotton plant and allowed to lay eggs with 10 replicates for each treatment. After 7 days, egg numbers were recorded.

\section{Amino acid sequence alignment and phylogenetic tree analysis}

Amino acid sequence alignments were conducted among $B$. tabaci MEAM1 [17], MED [51, 52], SSA-ECA, and Asia II 
3 [53] and Trialeurodes vaporariorum and Halmiltonella using BioEdit v7.1.3.0. To construct the molecular phylogenetic tree for each of whitefly bioA, bioD, and bioB, a Bayesian inference analysis was conducted as described previously [17]. For more details, see the Supplementary Text.

\section{Statistical analyses}

For the egg numbers of dsGFP, dsbioA, dsbioD, and dsbioB-fed whiteflies, the egg numbers and mortality of $\mathrm{ds} G F P$, dsbioA and dsbioA plus biotin-fed female whiteflies, and the OD values of the E. coli wild-type K-12, mutant K-12 and mutant K-12 transformants, statistical differences were evaluated using a one-way ANOVA at a significance threshold of 0.05 followed by LSD post-hoc tests. For the symbiont titer, biotin amount, gene expression level and mortality of $\mathrm{ds} G F P$ and $\mathrm{ds} b i o A, \mathrm{ds} b i o D$ and ds $b i o B$-fed female whiteflies, statistical differences were evaluated using a one-way ANOVA at a significance threshold of 0.05 level. Data in proportions were transformed by arcsine square root before analysis. All data analyses were conducted using the STATISTICA v6.1 software (StatSoft, Inc., Tulsa, OK, USA).

\section{Results}

\section{Hamiltonella elimination decreases biotin levels and elevates expression of horizontally transferred biotin genes in whiteflies}

The whitefly B. tabaci MEAM1 bears Portiera and Hamiltonella in the same bacteriocyte and Rickettisa in the whole body cavity, particularly in the abdomen (Fig. 1a, b). To investigate whether Hamiltonella contributes to the generation of biotin, Hamiltonella was eliminated by $86 \%$ through treating whitefly adults with a cocktail of antibiotics (Fig. 1a, c) $(P<0.05$ for Hamiltonella). Both FISH and qPCR revealed that Hamiltonella elimination didn't influence the abundance of Portiera and Rickettisa (Fig. 1a-c) $(P>0.05$ for Portiera and Rickettisa). Hamiltonella elimination decreased biotin level in whiteflies over time after antibiotic treatment
Fig. 1 Effects of Hamiltonella elimination on the biotin levels and HTG expression in whiteflies. a Localization of symbiotic bacteria Portiera (red) and Hamiltonella (green) in adult whiteflies. b Localization of symbiotic bacteria Portiera (red) and Rickettsia (blue) in adult whiteflies. c Effects of antibiotic treatments on the abundance of symbionts in whiteflies. d Effects of Hamiltonella elimination on biotin levels in whiteflies within 0-7 days after emergence at the F1 stage. e Genetic duplication of biotin synthesis for the whitefly B. tabaci HTGs and symbiont Hamiltonella (This figure is adapted from previous work [17, 42]). f Effect of Hamiltonella elimination on HTG expression in whiteflies. $+\mathrm{HBt}$ and $-\mathrm{HBt}$ represent Hamiltonella-infected and Hamiltonella-cured whiteflies, respectively. Data shown are mean \pm SE. Different letters above the bars indicate significant differences between treatments at $P<0.05$.

\section{A}

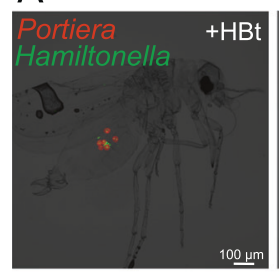

C

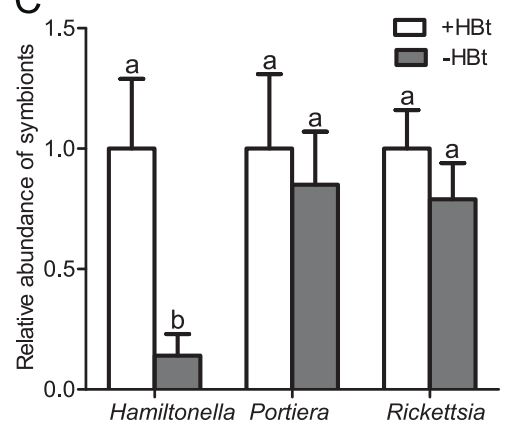

E
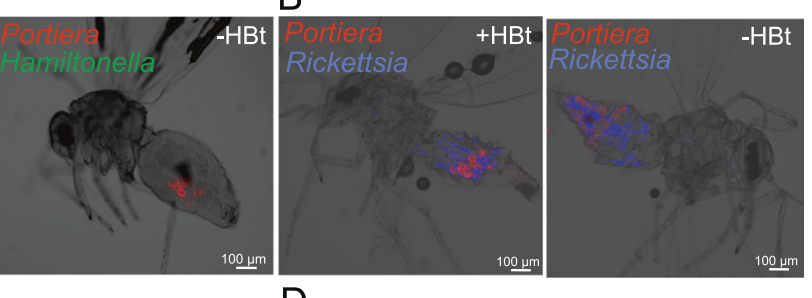

D

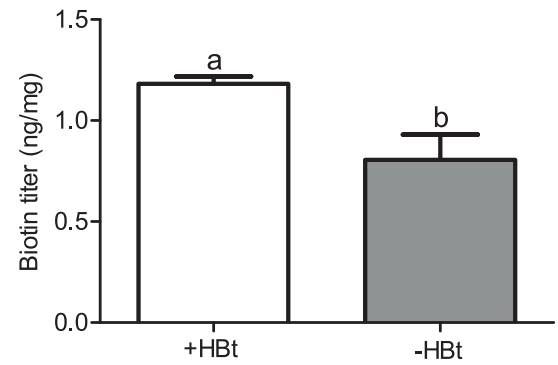

$\mathrm{F}$
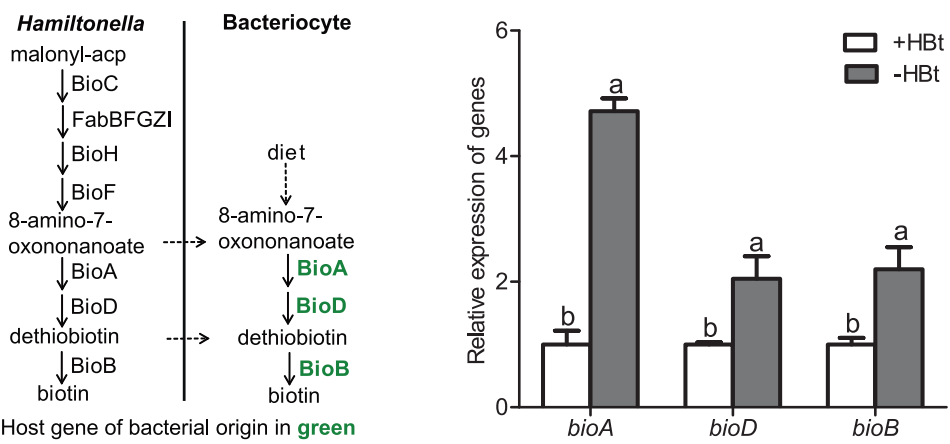
(Fig. 1d; $P<0.01$ and Supplementary Fig. $1 ; P<0.05$ ). Our earlier work showed that there is redundancy in biotin biosynthesis from both whitefly and Hamiltonella because of the presence of whitefly HTGs [17, 42] (Fig. 1e). Interestingly, after Hamiltonella was eliminated, expression of horizontally transferred bioA, bioD or bioB was significantly elevated (Fig. 1f) $(P<0.05)$, indicating that horizontally transferred biotin genes are functional in the biotin synthesis of whiteflies. In addition, we found that Rickettisa genome has lost key genes (e.g., bioA, bioD, and $b i o B$ ) involved in biotin synthesis (Supplementary Table 2) [42]. So Rickettisa is not able to synthesize biotin itself and the role of Rickettsia in biotin provisioning in this whitefly strain can be excluded.

\section{Horizontally transferred biotin synthesis proteins have specific expression patterns in whitefly bacteriocytes}

To examine the subcellular location of the proteins encoded by whitefly horizontally transferred $b i o A, b i o D$, or bioB in bacteriocytes, the recombinant proteins were successfully generated (Supplementary Fig. 2a-c). Then, polyclonal antibodies against $\mathrm{BioA}, \mathrm{BioD}$, and $\mathrm{BioB}$ proteins were produced using the purified recombinant protein. Polyclonal antibodies showed consistent specificity to those proteins, which was verified by western blot (Supplementary Fig. 2d-f). Hamiltonella was mainly distributed around bacteriocyte nuclei and Portiera occupied the cytoplasmic regions of bacteriocytes [40] (Fig. 2a). Immunofluorescence microscopy showed that BioA and BioD were mainly located in the peripheral regions of bacteriocytes in contact with external medium (Fig. 2b, c), while BioB was distributed both in the peripheral regions and around bacteriocyte nuclei in whiteflies infected with Hamiltonella (Fig. 2d). After Hamiltonella was cured, the protein expression levels and patterns were maintained in whiteflies (Supplementary Fig. 3), confirming that $\mathrm{BioA}, \mathrm{BioD}$, and $\mathrm{BioB}$ were not encoded by Hamiltonella. In contrast, no or a very weak signal was detected for these proteins in the guts as the control (Fig. 2e), where there were abundant Rickettsia as reported previously [54], indicating that $\mathrm{BioA}, \mathrm{BioD}$, and BioB were not encoded by Rickettsia, too. These data further suggest that BioA, BioD and BioB likely perform some functions in bacteriocytes.

\section{Functional complementation of $E$. coli biotin auxotrophs with whitefly biotin genes}

To test the hypothesis that bioA, bioD and bioB of the whitefly function in biotin synthesis, the $E$. coli $\mathrm{K}-12$ bioA, bioD and bioB knockout mutant $(-\Delta b i o A,-\Delta b i o D$, or
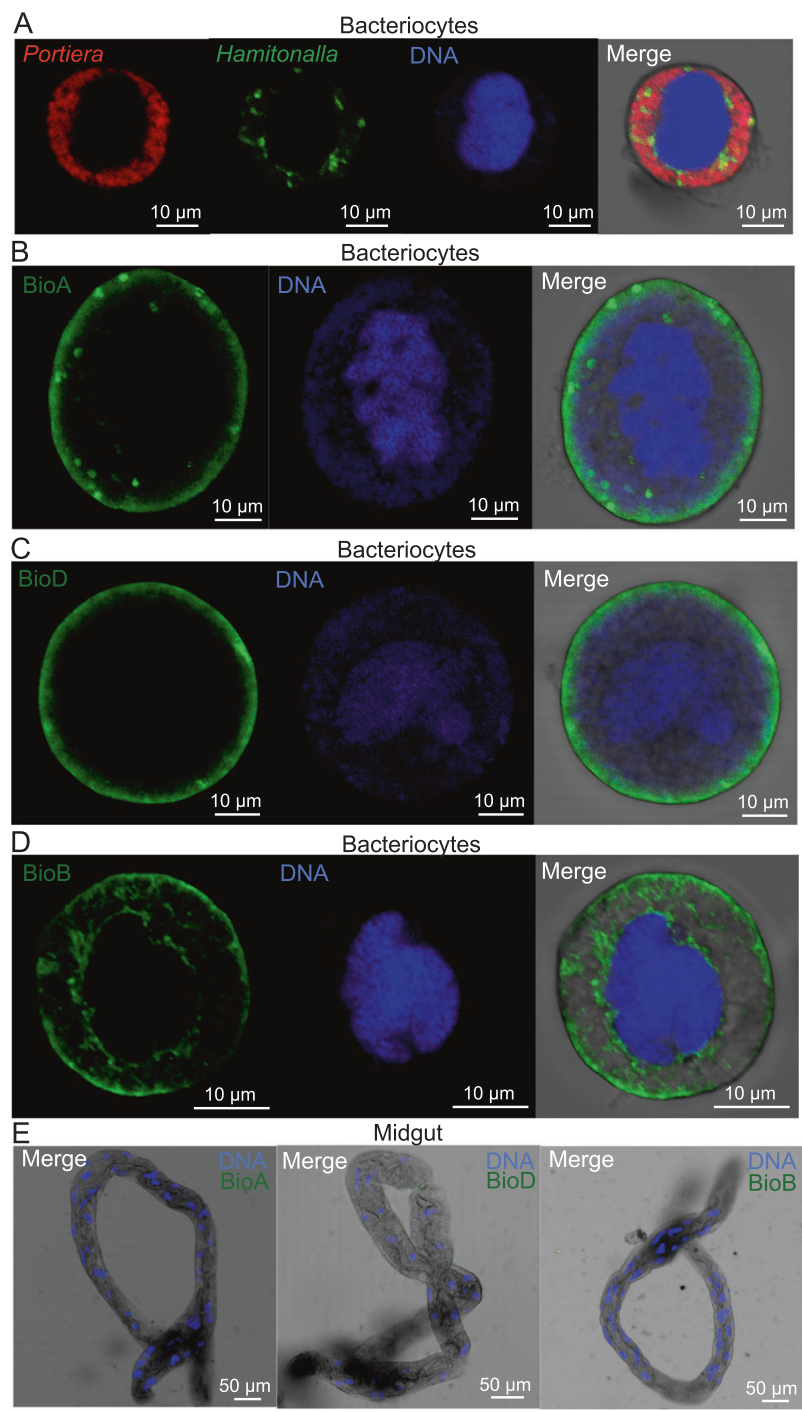

Fig. 2 Localization of horizontally transferred biotin synthesis proteins in whiteflies. a Localization of symbiotic bacteria Portiera (red) and Hamiltonella (green) in the bacteriocytes of adult whiteflies. Localization of BioA, BioD, and BioB proteins (green) in the bacteriocytes (b-d) and guts (e) of female adult whiteflies. DNA was stained with DAPI.

$-\triangle b i o B)$ were generated using the Lambda Red protocol and functionally complemented $E$. coli K-12 mutant with whitefly bioA, $\operatorname{bio} D$, and $\operatorname{bio} B$, respectively. Compared with wild-type E. coli, E. coli K-12 knockout mutants (- $\Delta b i o A$, $-\triangle b i o D$, and $-\triangle$ bioB) grew poorly on LB media and did not grow on B7DB media lacking biotin (Fig. 3a-d). Significant differences in OD values among treatments were detected (Fig. 3a; $P<0.001$ for bioA, bioD, and bioB). Although whitefly bioA, bioD, and $\operatorname{bioB}$ shared low amino acid sequence similarities with $E$. coli homolog genes $(34.85 \%$, $23.5 \%$, and $50.87 \%$, respectively) (Supplementary Fig. $4 \mathrm{a}-\mathrm{c}$ ), complementation with whitefly bioA, bioD and bioB rescued $E$. coli $\mathrm{K}-12$ knockout mutants on $\mathrm{LB}$ and B7DB media (Fig. 3a-d). In contrast, cells transformed with 
A
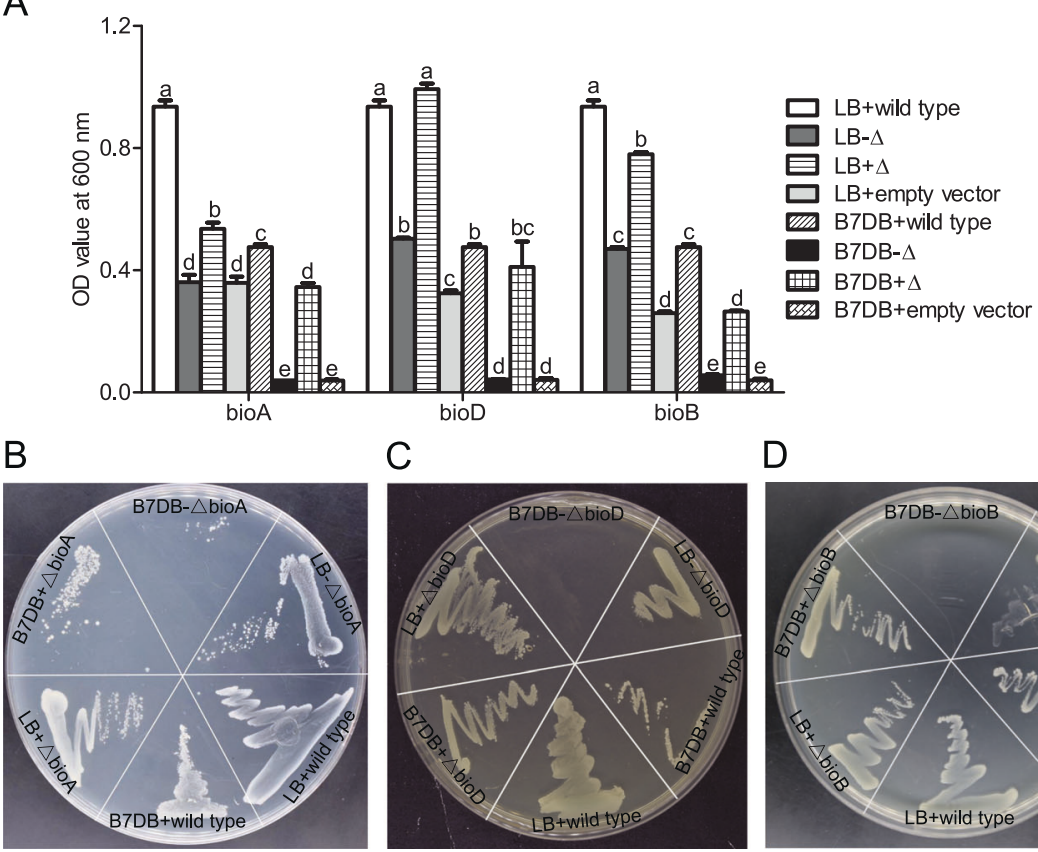

C
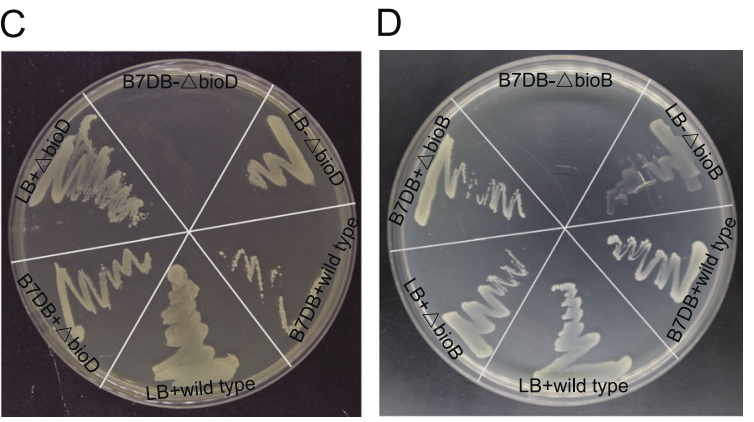

Fig. 3 Functional complementation of $\boldsymbol{E}$. coli biotin auxotrophs. a $E$. coli $\mathrm{K}-12$ knockout mutant cells were transformed with expression plasmids containing whitefly bioA, bioD, bioB or the negative control pMD19-T empty vector. The $E$. coli wild-type K-12, mutant $\mathrm{K}-12(-\Delta)$ and mutant K-12 transformants $(+\Delta)$ were grown overnight in LB and vitamin B7-deficient Difco biotin assay media (named as B7DB) at $37^{\circ} \mathrm{C}$. All E. coli cells were washed and resuspended to

the pMD19-T empty vector grew poorly on LB media and did not grow on B7DB media without biotin supplementation (Fig. 3a-d).

\section{Silencing horizontally transferred biotin genes reduces biotin levels and whitefly fitness while biotin supplementation restores whitefly performance}

To confirm the metabolic function of horizontally transferred biotin genes, the gene silencing approach was applied in Hamiltonella-infected whiteflies. Expression of whitefly bioA, bioD, and bioB were reduced by $70-94 \%$ after feeding on dsRNAs for 3 days (Fig. $4 \mathrm{a}-\mathrm{c})(P<0.01$ for bioA; $P<0.01$ for bioD and $P<0.001$ for bioB). In addition, the protein expression levels of $\mathrm{BioA}, \mathrm{BioD}$, and $\mathrm{BioB}$ were significantly reduced in bacteriocytes after the RNAi treatment of these whiteflies (Fig. 4d-f). Silencing whitefly biotin genes significantly reduced biotin levels (Fig. $4 \mathrm{~g}-\mathrm{i}$ ) $(P<0.0001$ for bioA; $P<0.05$ for bioD and $P<0.001$ for bio $B)$. As a result, gene silencing significantly increased the mortality of female adult whiteflies after 3 days, except for bioD silencing after day 2 and 3 (Fig. 4j-1) $(P<0.05$ for bioA and $b i o B$ at day 1,2 , and $3 ; P<0.001$ for bioD at day 1 and $P>0.05$ for $b i o D$ at day 2 and 3). After biotin measure cell density at $\mathrm{OD}_{600}$. E. coli wild-type K-12, mutant K-12 and mutant K-12 transformants were plated on LB and B7DB media agar plates for bioA (b), for bioD (c) and for bioB (d). Recovery of $E$. coli cell growth was assessed after overnight incubation at $37^{\circ} \mathrm{C}$. Data shown are mean $\pm \mathrm{SE}$. Different letters above the bars indicate significant differences between treatments at $P<0.05$.

supplementation in the artificial diet, the mortality of dsbioA-fed whiteflies was decreased over 3 days, which is close to that of dsGFP-fed whiteflies (Fig. $4 \mathrm{~m})(P>0.05$ for day 1 and $2 ; P<0.05$ for day 3$)$. In addition, gene silencing significantly repressed the fecundity of female adult whiteflies (Fig. 4n) $(P<0.0001)$. Meanwhile, after biotin supplementation in the artificial diet, the fecundity of dsbioA-fed whiteflies was restored (Fig. 4o) $(P<0.0001)$. Furthermore, the abundance of Hamiltonella was not changed in RNAi treated whiteflies (Supplementary Fig. 5) $(P>0.05)$, suggesting that Hamiltonella could not complement the depleted roles of horizontally transferred biotin genes in whiteflies.

\section{Evolutionary origin of horizontally transferred biotin genes in whiteflies}

To investigate whether horizontally transferred biotin genes are ubiquitous in whitefly populations, the presence of bioA, bioD, and bioB was checked in multiple whitefly species and cultures. We found that all of nine whitefly cultures of five species, which are distributed in Asia, America, Europe and Africa, possess these horizontally transferred biotin genes (Supplementary Table 3). To examine the divergence of protein sequences, amino acid 
A

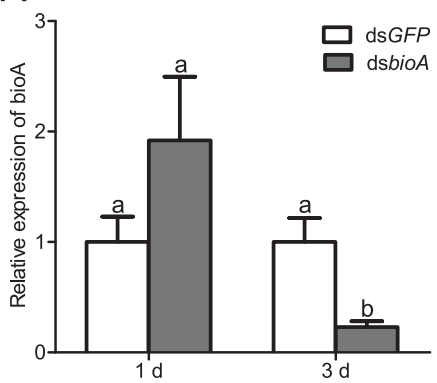

$\mathrm{D}$

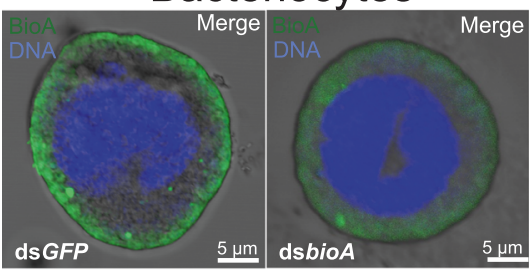

G

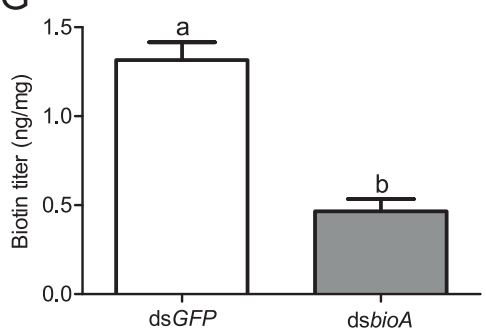

$\mathrm{J}$

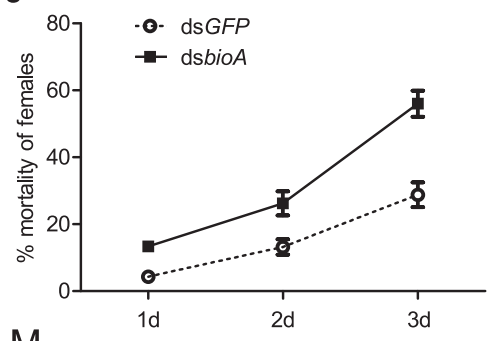

$\mathrm{M}$

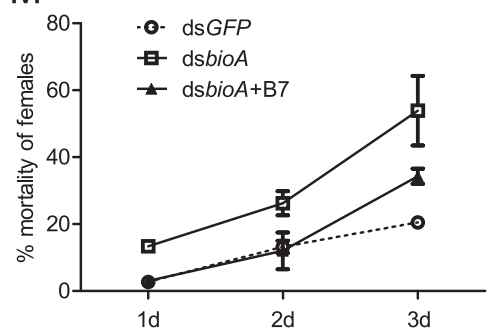

B

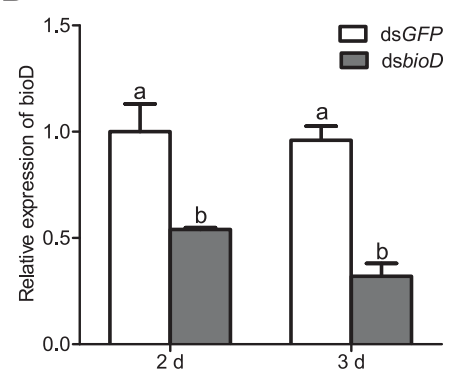

E Bacteriocytes

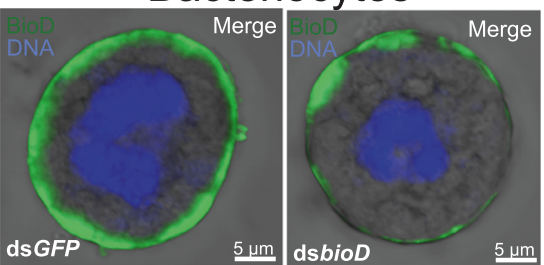

$\mathrm{H}$

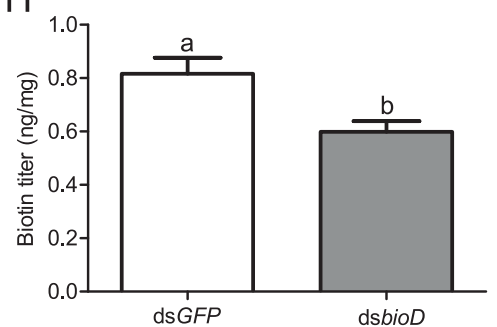

$\mathrm{K}$
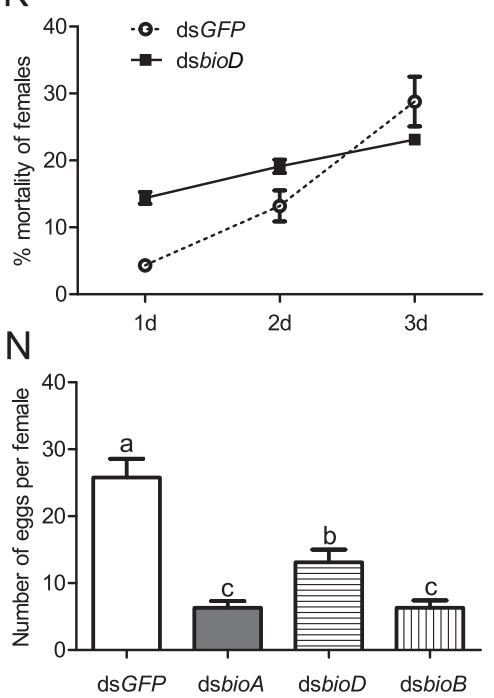

C

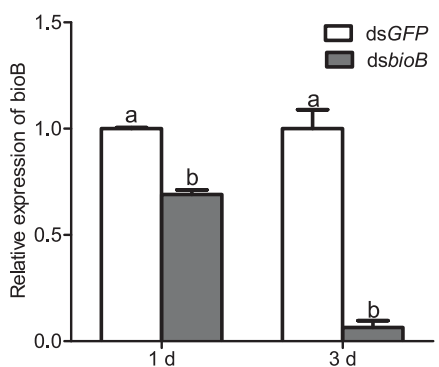

F Bacteriocytes

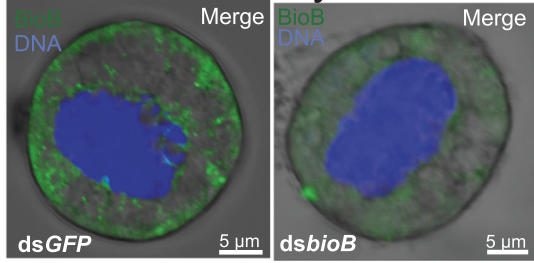

।

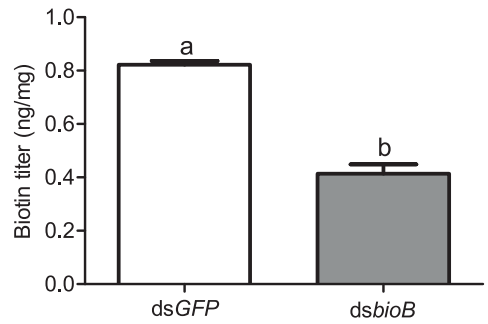

L
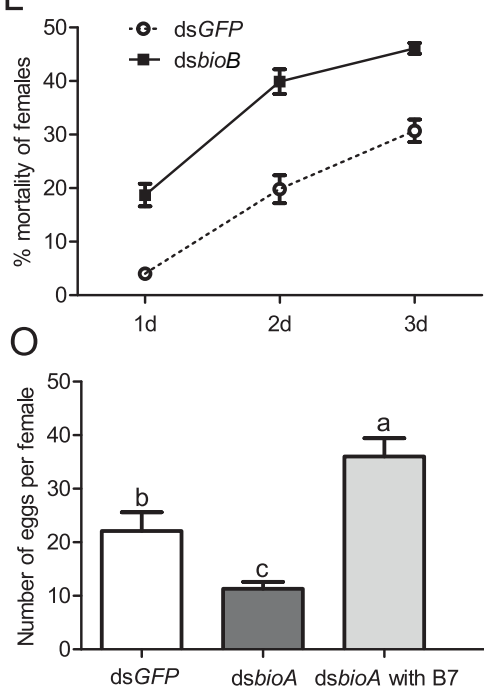

Fig. 4 Supplementation with biotin restores fitness of RNAi whiteflies. Expression of whitefly bioA (a), bioD (b), and bioB (c) after whiteflies fed on dsRNAs for 3 days. Localization of BioA (d), $\operatorname{BioD}(\mathbf{e})$, and BioB (f) proteins (green) in the bacteriocytes of female adult RNAi whiteflies. DNA was stained with DAPI. Biotin levels in whiteflies after feeding on dsbioA (g), dsbioD (h) and dsbioB (i) for 3 days. Mortality of female adult whiteflies after feeding on dsbioA (j), dsbioD (k), and dsbioB (l) for 3 days. m Mortality of female adult whiteflies after feeding on dsbioA and dsbioA plus B7 biotin for 3 days. n Fecundity of female adult whiteflies after feeding on dsRNAs for 3 days. o Fecundity of female adult whiteflies after feeding on dsbioA and dsbioA plus B7 biotin for 3 days. dsGFP-fed whiteflies were used as the control. Data shown are mean \pm SE. Different letters above the bars indicate significant differences between treatments at $P<0.05$.

species and Hamiltonella for bioB. The amino acid sequence identity was high among all whitefly species Hamiltonella for bioA and bioD and among 5 whitefly 


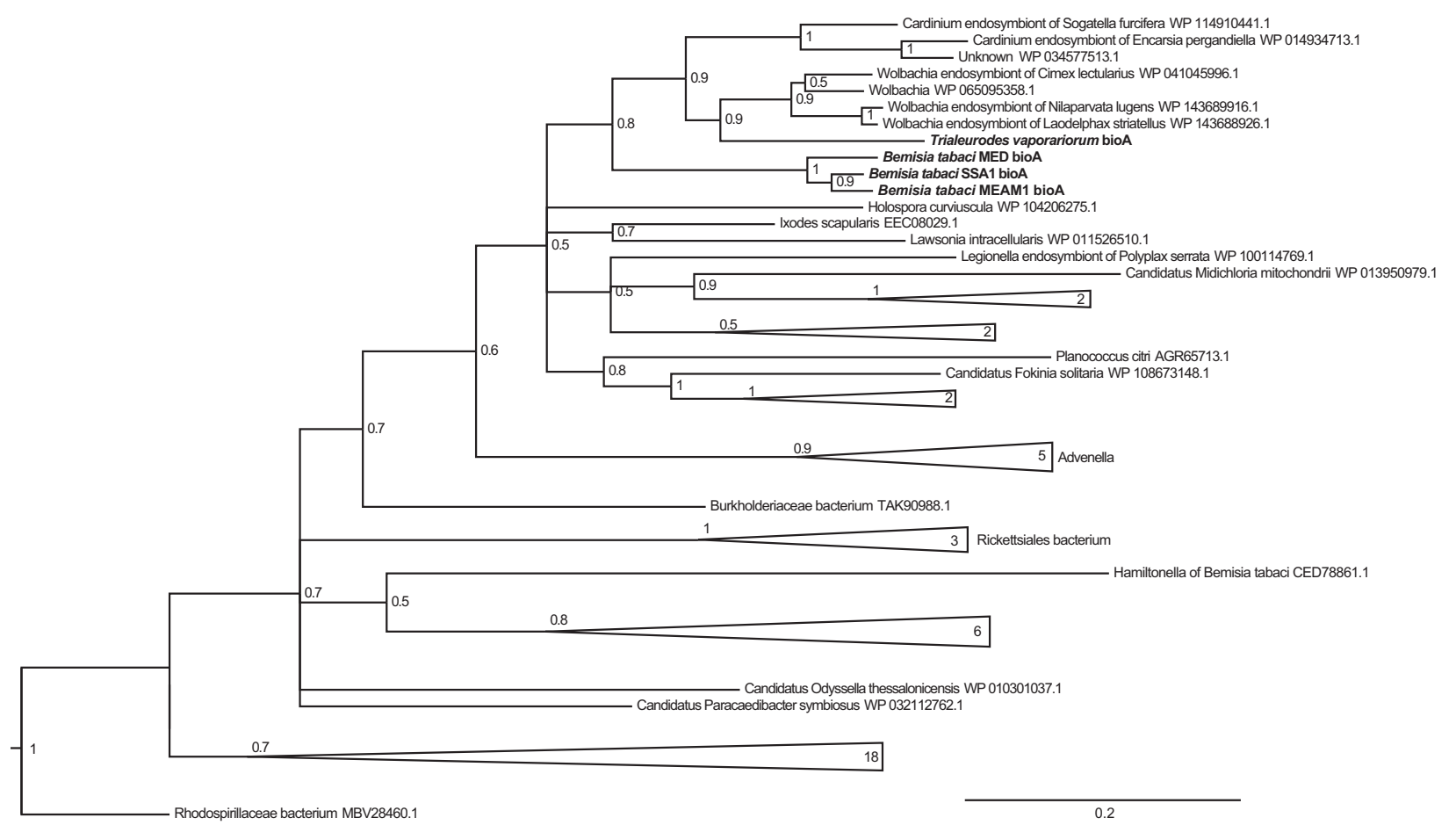

Fig. 5 Phylogenetic tree analysis of horizontally transferred BioA in the whitefly. Bayesian inference analysis was conducted for tree construction. A posterior probability of each node was shown.

(78-88\%) but low between whiteflies and Hamiltonella (25-48\%), indicating that bioA, bioD, and bioB were not horizontally transferred from Hamiltonella (Supplementary Fig. $4 \mathrm{a}-\mathrm{c}$ ). To gain insight into the evolution of these HTGs, a phylogenetic tree was constructed. Interestingly, BioA, BioD, and BioB of all whitefly species clustered within the same clade (Fig. 5; Supplementary Fig. 6a, b). Furthermore, whitefly BioA and BioD clustered with Wolbachia strains of the bedbug and planthoppers and Cardinium associated with the whitefly parasitioid and planthopper (Fig. 5; Supplementary Fig. 6a). BioB fell within the clade of Rickettsia, and clustered with Wolbachia strains of the bedbug and planthoppers and Cardinium associated with the whitefly parasitioid and planthopper (Supplementary Fig. 6b). Collectively, these data suggest that horizontally transferred BioA, BioD, and $\mathrm{BioB}$ share a common evolutionary origin in whiteflies.

\section{Discussion}

Using multi-disciplinary approaches, we have demonstrated that whiteflies are able to synthesize biotin via HTGs of bacterial origins. Because biotin is essential for whitefly fitness and horizontally transferred biotin synthesis genes are widespread in whiteflies, these results show that horizontal gene transfer contributes to the ability of whitefly to feed on B vitamin deficient phloem and so infest diverse crop plants. Our findings suggest that B vitamin provisioning in animal-microbe symbiosis frequently evolved from bacterial symbionts to animal hosts through horizontal gene transfer events.

Previously, the function of horizontally transferred biotin genes in whitefly and mealybug was speculated to be involved in biotin synthesis based on genomic and transcriptomic data [1, 17, 42]. Intron gain and duplication of the HTGs are thought to be the key steps when becoming functional in a eukaryotic genome [55]. In whiteflies, horizontally transferred $b i o B$ has acquired intron, and bioA (Bta01937 and Bta00841) and bioD (Bta00840 and Bta01938) were duplicated, of which, one of these duplicated genes (Bta00841 and Bta01938) was pseudogenized [42]. Our results support the hypothesis that intron gain and duplication of HTGs may help them become functional [55]. Interestingly, the elimination of Hamiltonella increased the expression of whitefly biotin genes. In addition, horizontally transferred $\mathrm{BioA}, \mathrm{BioD}$, and BioB proteins are enriched in bacteriocytes when compared with the guts. Localization in peripheral regions of bacteriocytes may help BioA and BioD access to precursors or intermediate metabolites from hemolymph for biotin synthesis. In contrast, distribution in the peripheral regions and around bacteriocyte nuclei could allow BioB to utilize dethiobiotin, the product of reaction mediated by $\mathrm{BioD}$, from either HTGs or Hamiltonella, which mainly distributed around 
bacteriocyte nuclei [40]. Cell-specific expression patterns of these proteins strongly suggest that they are functional. Furthermore, complementation with whitefly HTGs restored E. coli biotin gene knockout mutants, indicating that whitefly biotin genes serve the same function as $E$. coli homolog genes. Indeed, silencing whitefly HTGs reduced biotin levels. Biotin is the coenzyme of carboxylases and plays critical roles in central metabolic processes [21]. Not surprisingly, silencing whitefly HTGs hindered insect performance dramatically, which was restored by dietary biotin supplementation. To our knowledge, this study is the first to present empirical evidence that although Hamiltonella are able to produce biotin, whiteflies are able to synthesize biotin through acquired HTGs. A recent study shows that HTGs in a mealybug cooperate with genes of the bacterial symbiont Moranella to produce the peptidoglycan layer at the Moranella cell periphery [20]. These findings provide excellent genetic targets for insect control by selective interference.

There is redundancy in biotin synthesis from both whitefly and Hamiltonella. The biotin synthesized by Hamiltonella could be used for its growth and reproduction or provided to whiteflies. So, the importance of biotin and other B vitamins provisioned by Hamiltonella in whitefly fitness is worthy investigation in the future. This may explain why Hamiltonella appears to be a critical nutritional mutualist. In addition, the acquisition of biotin synthesis capability in whiteflies via HTGs may help whiteflies not to completely depend on the symbiont for biotin synthesis. Such metabolic redundancy reflects the coevolution of the host and symbiont.

Rickettsia can give fitness benefits in B. tabaci MEAM1 in USA [56], suggesting that this symbiont may synthesize biotin for the host. Because Hamiltonella elimination did not influence the abundance of Rickettisa, reduction of biotin level in Hamiltonella-cured whiteflies is not caused by changes of Rickettisa titer. Furthermore, Rickettisa genome has lost key biotin genes, revealing that Rickettisa is not able to synthesize biotin itself. Moreover, there is no possibility of non-target RNAi knockdowns for Rickettisa genes. Collectively, the role of Rickettsia in biotin provisioning in this whitefly strain can be excluded, and the results in this study are not due to the infection of these whiteflies with Rickettsia.

Notably, all nine whitefly cultures of the five species we tested harbored these horizontally transferred biotin genes. Among the five whitefly species, the whitefly $B$. tabaci MEAM1, MED, and T. vaporariorum are globally important pests in agriculture and the whitefly B. tabaci SSAECA and Asia II 3 are local pests found in Africa and Asia, respectively. It seems that the acquisition of horizontally transferred biotin genes is independent of whitefly species and their geographical region, suggesting a common evolutionary origin of these HTGs. The phylogenetic tree analysis revealed that BioA, BioD, and BioB cluster with Wolbachia, Cardinium, or Rickettsia. The whitefly B. tabaci MEAM1 harbors Rickettsia [57, 58], B. tabaci Asia II 3 bears Rickettsia and Cardinium [57], and B. tabaci MED and T. vaporariorum harbor Rickettsia, Cardinium and Wolbachia [59-61]. Therefore, HTGs were likely transferred to the common ancestor of whiteflies from Rickettsia, Cardinium or Wolbachia before the divergence of whiteflies infected with these symbionts. This hypothesis is supported by the fact that biotin synthesis genes are absent in the genomes of Rickettsia, Cardinium, and Wolbachia (accession No.: CP016430.1) associated with whiteflies [42, 61]. In addition, BioA, BioD, and BioB were very closely related to Cardinium associated with the whitefly parasitoid Encarsia pergandiella that is widely distributed in the world [62, 63]. Therefore, Cardinium likely switched hosts between the whitefly and parasitoid early in their evolution and then independently diverged in two hosts. Last, biotin genes were lost in Cardinium and transferred to the whitefly host. The ubiquity of horizontally transferred biotin genes in various whitefly populations indicates that they are essential for whitefly evolution, which may lead to the loss of biotin synthesis genes in whitefly symbionts, including Hamiltonella. This speculation is supported by the fact that biotin synthesis pathway of Moranella is degenerated in the mealybug, which bears horizontally transferred biotin genes of Rickettsiales origin [1] and also bioH, bioA and bioD of Arsenophonus [32] are pseudogenes in the whitefly $T$. vaporariorum which possesses horizontally transferred bioA, bioD, and bioB.

Our findings along with previous studies present three modes of B vitamin provisioning in insect symbiosis. The first is that symbionts such as Francisella and Wigglesworthia provide B vitamins including biotin for ticks and tsetse flies without being related to HTGs [23-27]. The second is that symbionts provide B vitamins for insects in bedbug/planthopper-Wolbachia symbiosis and potentially in aphid-Erwinia symbiosis through acquired HTGs for B vitamin synthesis [2, 22, 33, 36]. The biotin operon of Wolbachia is acquired via horizontal gene transfer, presumably from coinfecting Cardinium or Rickettsia in bedbugs [2] and from Cardinium in planthoppers [36], and bioA, bioD and bioB of Erwinia are horizontally transferred from Sodalis or Sodalis-like bacteria [33]. The results of this study uncovered a third mechanism in that both symbiont and insect HTGs synthesize biotin, but biotin synthesized by whitefly HTGs with bacteria origin determines insect performance. Interestingly, whitefly BioA, BioD and BioB clustered with Wolbachia strains of the bedbug and planthoppers, indicating that horizontal gene transfer events of bacteria-to-insect and bacteria-to-Wolbachia shares a common biological and evolutionary histories $[2,36]$. In 
addition, whitefly BioA, BioD, and BioB clustered with closely related mealybugs of the same Sternorrhyncha, suggesting that mealybugs also have biotin synthesis potential through horizontally transferred biotin genes [1]. Collectively, three mechanisms summarized here all point to the convergent role of biotin in insect symbiosis. Thus, this study suggests that the transition of B vitamins provisioned by symbionts to insect hosts is mediated through horizontal gene transfer. It will be worth investigating the role of HTGs in the synthesis of B vitamins in diverse insect symbiosis systems in the future.

\section{Data availability}

All relevant data supporting the findings of this study are included within the article and its Supplementary Information files.

Acknowledgements We would like to thank Professor Myron Zalucki of the University of Queensland, Australia for his constructive comments and suggestions on the paper. The authors thank Professor Liu Shu-Sheng for providing the whitefly B. tabaci MEAM1 culture, and Dr. Lu Gang, Bao Xi-Yu, Li Ce, Li Chu-Qiao, and Wang Yan-Bin for their assistance with the experiments. This work was supported by the National Natural Science Foundation of China (No. 31871967), HighTech R\&D Program of Liaoning (No. 2019JH2/10200012), and HighLevel Talent Support Foundation from Liaoning, Shenyang and Shenyang Agricultural University (Project XLYC1902104, RC180025 and 880418001).

Author contributions JBL conceived the study. FRR conducted symbiont elimination, biotin assays, ecology experiments, gene expression analyses, and gene silencing. FRR, XS, TYW, and XZ performed the complementation experiments. XS carried out gene silencing, FISH and immunofluorescence experiments. YLY constructed the phylogenetic tree. YZH helped design the biotin assays. FRR, JBL, and YZH analyzed the data. JBL wrote the manuscript. All authors edited and approved the final manuscript.

\section{Compliance with ethical standards}

Conflict of interest The authors declare that they have no conflict of interest.

Publisher's note Springer Nature remains neutral with regard to jurisdictional claims in published maps and institutional affiliations.

\section{References}

1. Husnik F, Nikoh N, Koga R, Ross L, Duncan RP, Fujie M, et al. Horizontal gene transfer from diverse bacteria to an insect genome enables a tripartite nested mealybug symbiosis. Cell. 2013;153:1567-78.

2. Nikoh N, Hosokawa T, Moriyama M, Oshima K, Hattori M, Fukatsu T. Evolutionary origin of insect-Wolbachia nutritional mutualism. Proc Natl Acad Sci USA. 2014;111:10257-62.

3. Salem H, Bauer E, Kirsch R, Berasategui A, Cripps M, Weiss B, et al. Drastic genome reduction in an herbivore's pectinolytic symbiont. Cell. 2017;171:1520-31.
4. Moran NA, McCutcheon JP, Nakabachi A. Genomics and evolution of heritable bacterial symbionts. Annu Rev Genet. 2008;42:165-90.

5. Moya A, Peretó J, Gil R, Latorre A. Learning how to live together: genomic insights into prokaryote-animal symbioses. Nat Rev Genet. 2008;9:218-29.

6. Douglas AE. Multiorganismal insects: diversity and function of resident microorganisms. Annu Rev Entomol. 2015;60:17-34.

7. Moran NA, Bennett GM. The tiniest tiny genomes. Annu Rev Microbiol. 2014;68:195-215.

8. Beiko RG, Harlow TJ, Ragan MA. Highways of gene sharing in prokaryotes. Proc Natl Acad Sci USA. 2005;102:14332-7.

9. Wybouw N, Pauchet Y, Heckel DG, Van Leeuwen T. Horizontal gene transfer contributes to the evolution of arthropod herbivory. Genome Biol Evol. 2016;8:1785-801.

10. Dunning Hotopp JC, Clark ME, Oliveira DCSG, Foster JM, Fischer P, Torres MCM, et al. Widespread lateral gene transfer from intracellular bacteria to multicellular eukaryotes. Science. 2007;317:1753-6.

11. Dunning Hotopp JC. Horizontal gene transfer between bacteria and animals. Trends Genet. 2011;27:157-63.

12. Moran NA, Jarvik T. Lateral transfer of genes from fungi underlies carotenoid production in aphids. Science. 2010;328:624-7.

13. Bryon A, Kurlovs AH, Dermauw W, Greenhalgh R, Riga M, Grbic M, et al. Disruption of a horizontally transferred phytoene desaturase abolishes carotenoid accumulation and diapause in Tetranychus urticae. Proc Natl Acad Sci USA. 2017;114:5871-80.

14. Acuña R, PadillaB E, Claudia P, Flórez-Ramos José D, Rubio Herrera JC, Benavides P, et al. Adaptive horizontal transfer of a bacterial gene to an invasive insect pest of coffee. Proc Natl Acad Sci USA. 2012;109:4197-202.

15. Wybouw N, Dermauw W, Tirry L, Stevens C, Grbic M, Feyereisen $\mathrm{R}$, et al. A gene horizontally transferred from bacteria protects arthropods from host plant cyanide poisoning. eLife. 2014;3: E02365.

16. Sloan DB, Nakabachi A, Richards S, Qu J, Murali SC, Gibbs RA, et al. Parallel histories of horizontal gene transfer facilitated extreme reduction of endosymbiont genomes in sap-feeding insects. Mol Biol Evol. 2014;31:857-71.

17. Luan JB, Chen WB, Hasegawa DK, Simmons AM, Wintermantel $\mathrm{WM}$, Ling KS, et al. Metabolic coevolution in the bacterial symbiosis of whiteflies and related plant sap-feeding insects. Genome Biol Evol. 2015;7:2635-47.

18. Nakabachi A, Ishida K, Hongoh Y, Ohkuma M, Miyagishima SY. Aphid gene of bacterial origin encodes a protein transported to an obligate endosymbiont. Curr Biol. 2014;24:640-1.

19. Chung SH, Jing X, Luo Y, Douglas AE. Targeting symbiosisrelated insect genes by RNAi in the pea aphid-buchnera, symbiosis. Insect Biochem Mol Biol. 2018;95:55-63.

20. Bublitz DC, Chadwick GL, Magyar JS, Sandoz KM, Brooks DM, Mesnage S, et al. Peptidoglycan production by an insect-bacterial mosaic. Cell. 2019;179:703-12.

21. Douglas AE. The B vitamin nutrition of insects: the contributions of diet, microbiome and horizontally acquired genes. Curr Opin Insect Sci. 2017;23:65-69.

22. Hosokawa T, Koga R, Kikuchi Y, Meng XY, Fukatsu T. Wolbachia as a bacteriocyte-associated nutritional mutualist. Proc Natl Acad Sci USA. 2010;107:769-74.

23. Snyder AK, Deberry JW, Runyen-Janecky L, Rio RV. Nutrient provisioning facilitates homeostasis between tsetse fly (Diptera: Glossinidae) symbionts. Proc Biol Sci. 2010;277:2389-97.

24. Snyder AK, Rio RV. "Wigglesworthia morsitans" Folate (Vitamin B9) biosynthesis contributes to tsetse host fitness. Appl Environ Microbl. 2015;81:5375-86. 
25. Michalkova V, Benoit JB, Weiss BL, Attardo GM, Aksoy S. Vitamin B6 generated by obligate symbionts is critical for maintaining proline homeostasis and fecundity in tsetse flies. Appl Environ Microbiol. 2014;80:5844-53.

26. Moriyama M, Nikoh N, Hosokawa T, Fukatsu T. Riboflavin provisioning underlies Wolbachia's fitness contribution to its insect host. MBio. 2015;6:1732-47.

27. Duron O, Morel O, Noël V, Buysse M, Binetruy F, Lancelot R, et al. Tick-bacteria mutualism depends on $\mathrm{B}$ vitamin synthesis pathways. Curr Biol. 2018;28:1896-902.

28. Wu D, Daugherty SC, Van Aken SE, Pai GH, Watkins KL, Khouri $\mathrm{H}$, et al. Metabolic complementarity and genomics of the dual bacterial symbiosis of sharpshooters. PLoS Bio. 2006;4:e188.

29. Mccutcheon JP, Moran NA. Parallel genomic evolution and metabolic interdependence in an ancient symbiosis. Proc Natl Acad Sci USA. 2007;104:19392-7.

30. McCutcheon JP, McDonald BR, Moran NA. Convergent evolution of metabolic roles in bacterial co-symbionts of insects. Proc Natl Acad Sci USA. 2009;106:15394-9.

31. Rao Q, Wang S, Su YL, Bing XL, Liu SS, Wang XW. Draft genome sequence of "Candidatus Hamiltonella defense" an endosymbiont of the whitefly Bemisia tabaci. J Bacteriol. 2012;194:3558.

32. Santos-Garcia D, Juravel K, Freilich S, Zchori-Fein E, Latorre A, Moya A, et al. To B or not to B: comparative genomics suggests Arsenophonus as a source of B vitamins in whiteflies. Front Microbiol 2018;9:2254-70.

33. Manzano-Marín A, Coeur d'acier A, Clamens AL, Orvain C, Cruaud C, Barbe V, et al. Serial horizontal transfer of vitamin-biosynthetic genes enables the establishment of new nutritional symbionts in aphids' di-symbiotic systems. ISME J. 2020;14:259-73.

34. Price DR, Wilson AC. A substrate ambiguous enzyme facilitates genome reduction in an intracellular symbiont. BMC Biol. 2014;12:110-9.

35. Salem H, Bauer E, Strauss AS, Vogel H, Marz M, Kaltenpoth M. Vitamin supplementation by gut symbionts ensures metabolic homeostasis in an insect host. Proc Biol Sci. 2014;281:1838-48.

36. Ju J, Bing XL, Zhao D, Guo Y, Xi Z, Hoffmann AA, et al. Wolbachia supplement biotin and riboflavin to enhance reproduction in planthoppers. ISME J. 2019;14:1-12.

37. Brown JK, Frohlich DR, Rosell RC. The sweetpotato or silverleaf whiteflies: biotypes of Bemisia tabaci or a species complex? Annu Rev Entomol. 1995;40:511-34.

38. Liu SS, De Barro PJ, Xu J, Luan JB, Zang LS, Ruan YM, et al. Asymmetric mating interactions drive widespread invasion and displacement in a whitefly. Science. 2007;318:1769-72.

39. De Barro PJ, Liu SS, Boykin LM, Dinsdale AB. Bemisia tabaci: a statement of species status. Annu Rev Entomol. 2011;56:1-19.

40. Luan JB, Shan HW, Isermann P, Huang JH, Lammerding J, Liu SS, et al. Cellular and molecular remodelling of a host cell for vertical transmission of bacterial symbionts. Proc R Soc B. 2016;283:20160580.

41. Luan JB, Sun XP, Fei ZJ, Douglas AE. Maternal inheritance of a single somatic animal cell displayed by the bacteriocyte in the whitefly Bemisia tabaci. Curr Biol. 2018;28:459-65.

42. Chen W, Hasegawa DK, Kaur N, Kliot A, Pinheiro PV, Luan JB, et al. The draft genome of whitefly Bemisia tabaci MEAM1, a global crop pest, provides novel insights into virus transmission, host adaptation, and insecticide resistance. Bmc Biol. 2016;14:110.

43. Schmittgen TD, Livak KJ. Analyzing real-time PCR data by the comparative CT method. Nat Protoc. 2008;3:1101-8.

44. Ren FR, Bai B, Hong JS, Huang YZ, Luan JB. A microbiological assay for biotin determination in insects. Insect Sci. 2020; https:// doi.org/10.1111/1744-7917.12827.
45. Shan HW, Luan JB, Liu YQ, Douglas AE, Liu SS. The inherited bacterial symbiont Hamiltonella influences the sex ratio of an insect host. Proc R Soc B 2019;286:20191677.

46. Gottlieb Y, Ghanim M, Chiel E, Gerling D, Portnoy V, Steinberg $\mathrm{S}$, et al. Identification and localization of a Rickettsia sp. in Bemisia tabaci (Homoptera: Aleyrodidae). Appl Environ Microbiol. 2006;72:3646-52.

47. Russell CW, Bouvaine S, Newell PD, Douglas AE. Shared metabolic pathways in a coevolved insect-bacterial symbiosis. Appl Environ Microbiol. 2013;79:6117-23.

48. Datsenko KA, Wanner BL. One-step inactivation of chromosomal genes in Escherichia coli K-12 using PCR products. Proc Natl Acad Sci USA. 2000;97:6640-5.

49. Baba T, Ara T, Hasegawa M, Takai Y, Okumura Y, Baba M, et al. Construction of Escherichia coli K-12 in-frame, single-gene knockout mutants: the Keio collection. Mol Syst Biol. 2006;2:2006-8.

50. Mori H, Baba T, Yokoyama K, TakeuchiR, Nomura W, Makishi $\mathrm{K}$, et al. Identification of essential genes and synthetic lethal gene combinations in Escherichia coli K-12. Methods Mol Biol. 2015;1279:45-65.

51. Xie W, Chen C, Yang Z, Guo L, Yang X, Wang D, et al. Genome sequencing of the sweetpotato whitefly Bemisia tabaci MED/Q. Gigascience. 2017;6:1-7.

52. Wang XW, Luan JB, Li JM, Bao YY, Zhang CX, Liu SS, et al. De novo characterization of a whitefly transcriptome and analysis of its gene expression during development. BMC Genomics. 2010;11:400-11.

53. Wang XW, Zhao QY, Luan JB, Wang YJ, Yan GH, Liu SS, et al. Analysis of a native whitefly transcriptome and its sequence divergence with two invasive whitefly species. BMC Genomics. 2012;13:529-42.

54. Brumin M, Levy M, Ghanim M. Transovarial transmission of Rickettsia spp. and organ-specific infection of the whitefly Bemisia tabaci. Appl Environ Microbiol. 2012;78:5565-74.

55. Husnik F, Mccutcheon JP. Functional horizontal gene transfer from bacteria to eukaryotes. Nat Rev Microbiol. 2018;16:67-79.

56. Himler AG, Adachi-Hagimori T, Bergen JE, Kozuch A, Kelly SE, Tabashnik BE, et al. Rapid spread of a bacterial symbiont in an invasive whitefly is driven by fitness benefits and female bias. Science. 2011;332::254-6.

57. Bing XL, Ruan YM, Rao Q, Wang XW, Liu SS. Diversity of secondary endosymbionts among different putative species of the whitefly Bemisia tabaci. Insect Sci. 2013;20:194-206.

58. Gottlieb Y, Ghanim M, Gueguen G, Kontsedalov S, Vavre F, Fleury F, et al. Inherited intracellular ecosystem: symbiotic bacteria share bacteriocytes in whiteflies. FASEB J. 2008;22:2591-9.

59. Škaljac M, Zanic K, Ban SG, Kontsedalov S, Ghanim M. Coinfection and localization of secondary symbionts in two whitefly species. BMC Microbiol. 2010;10:142-57.

60. Škaljac M, Žanić K, Hrnčić S, Radonjić S, Perović T, Ghanim M, et al. Diversity and localization of bacterial symbionts in three whitefly species (Hemiptera: Aleyrodidae) from the east coast of the Adriatic Sea. Bull Entomol Res. 2013;103:48-59.

61. Santos-Garcia D, Rollat-Farnier PA, Beitia F, Zchori-Fein E, Vavre F, Mouton L, et al. The genome of Cardinium cBtQ1 provides insights into genome reduction, symbiont motility, and its settlement in Bemisia tabaci. Genome Biol Evol. 2014;6:1013-30.

62. Penz T, Schmitz-Esser S, Kelly SE, Cass BN, Muller A, Woyke T, et al. Comparative genomics suggests an independent origin of cytoplasmic incompatibility in Cardinium hertigii. PLoS Genet. 2012;8:e1003012.

63. Liu TX, Stansly PA, Gerling D. Whitefly parasitoids: distribution, life history, bionomics, and utilization. Annu Rev Entomol. 2015;60:273-92. 